\title{
Theory and contents of frictional mechanics
}

\author{
Ping HUANG, Qianqian YANG* \\ School of Mechanical and Automotive Engineering, South China University of Technology, Guangzhou 510640, China \\ Received: 18 September 2013 / Revised: 12 November 2013 / Accepted: 19 November 2013 \\ (C) The author(s) 2013. This article is published with open access at Springerlink.com
}

\begin{abstract}
In this paper, we first discuss the development of the field of tribology, and highlight some of the main problems encountered in this area, such as lack of systematicness, loose correlation, and inadequate focus on the microscopic perspective. Then, we provide basic formulas of frictional mechanics while considering the friction effect on classical mechanics formulae. In order to carry out the frictional mechanics analysis, we first classify the interface. According to the size analysis of surface films, the manufacturing roughness of the surface, the contact width, and the roller radius of the rolling contact bearing, frictional mechanics has the features of interface mechanics, while interfaces are classified based on the presence or absence of a medium. Based on the classification, we further analyze the pressure and frictional stress of sliding and rolling friction problems without a medium, such as a slider, wedge key, and V belt. We also analyze problems with a medium, such as journal and rolling contact bearings. By comparing these results with those of classical mechanics without considering friction, we see that (1) friction causes deviations in the result for classical mechanics which does not consider friction, and (2) if the frictional stress and normal pressure affect each other, their interaction should be considered simultaneously. Finally, we summarize the friction problems, namely, sliding and rolling, with and without a medium, and deformed and non-deformed. From our analysis, we propose two conclusions. First, the frictional mechanics problem is a deviation of the classical mechanics problem, and secondly, frictional stress and normal pressure influence each other.
\end{abstract}

Keywords: frictional mechanics; interface; normal pressure; frictional stress

\section{Introduction}

Tribology is an applied subject that involves the study of friction, lubrication, and wear between two relatively moving surfaces [1]. Frictional phenomena have been recognized for thousands of years, such as rubbing wood to generate a fire, the invention of wheels, and the use of animal grease for lubrication purposes, etc. [2-5]. In the 15th century, Leonardo Da Vinci began the systematic study of friction, and Coulomb proposed the classical friction laws based on previous studies, that is, the frictional force is proportional to the normal load [1-5]. Then, the molecular friction theory, electrostatic friction theory, and adhesion theory laid the foundation for modern

* Corresponding author: Qianqian YANG.

E-mail: qqyangscut@163.com friction theory. Modern lubrication studies began in 1886, when Reynolds derived the hydrodynamic lubrication formulas based on the findings obtained by Tower [6]. The study of wear commenced much later, and although many wear theories were proposed in the latter half of the 20th century, none of them have been widely accepted $[7,8]$. The combined study of friction, wear, and lubrication began in 1966 when Jost published his report. The report, which estimated that nearly $1 / 3-1 / 2$ of the energy worldwide was wasted because of friction and wear, attracted the attention of many persons, and subsequently, the study of tribology was gradually developed.

Because tribology covers such a wide range of topics and there is a theoretical system of tribology, some of the main problems remain unsolved. First, the research contents and methods are different [9-12] 
because tribology involves a combination of friction, wear, and lubrication, which all differ in terms of research contents and approach. Friction caused by roughness is closely related to elastic deformation, so that contact mechanics is its main foundation. With respect to wear, a broken material is often considered, and it is based on material science and fracture mechanics. With respect to hydrodynamic lubrication, fluid mechanics is used to analyze the formation and performance of the lubrication film, and some of its research contents and methods are also based on subjects such as chemistry and physical chemistry. Therefore, the different research contents and methods relate not only to the characteristics of the three branches, but also to many other subjects. Another feature of tribology problems is their complexity. Because the formation and working mechanisms of friction, wear, and lubrication are complicated, no single theory can adequately explain these phenomena. Therefore, most tribological problems to be solved are related to solid mechanics, fluid mechanics, heat transmission, and chemistry, etc. [13-15].

Surface contact is very important in friction mechanics. Hertz solved the classical contact problem and developed contact mechanics. According to his study, after the loading of line contacts and point contacts, surfaces will deform to form a very small contact area. The Hertzian stress $\sigma_{\mathrm{H}}$ is used to determine the stress on the surfaces. For contact between two cylinders, the Hertzian stress $\sigma_{\mathrm{H}} \propto \sqrt{W}$, while for contact between two balls, $\sigma_{\mathrm{H}} \propto \sqrt[3]{W}$. The results indicate that pressure is not linearly proportional to the load $W$, as is experienced in common frictional problems involving contact between surfaces [16].

Moreover, a macroscopic study of tribology is considered to be not sufficient. Generally, the basic approach used to solve tribology problems has been to take a microscopic approach [9-19]. In 1932, Bradley provided an adhesive force calculation equation for two rigid balls to consider the adhesive work between two surfaces [20]. However, in his model, the objects were considered to be rigid. Then, Johnson, Kendal, and Roberts presented a stricter theory to analyze the contact between elastic balls, which is known as the JKR adhesive theory [21]. In their theory, a more significant result was that they gave a critical negative force $F_{\mathrm{ad}}$ which must be applied to separate the two surfaces. In 1975, Derjaguin, Muller, and Toprov put forward an equation known as the DMT adhesive contact model, which can be used to consider the relationship between the contact radius, load, and adhesive force [22]. These models are shown in Fig. 1. (a)

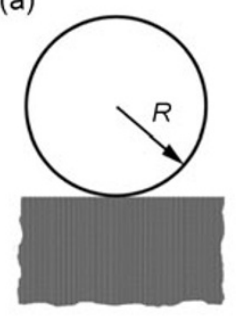

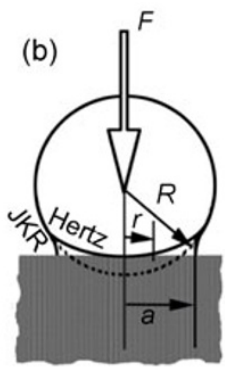

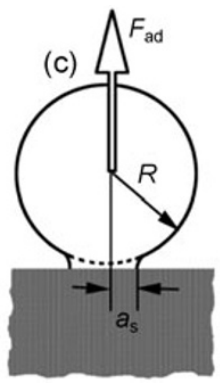

Fig. 1 Three different contact models of ball-plane contact [17]: (a) Rigid contact; (b) Hertz contact or JKR contact; (c) adhesive contact.

Although a microscopic analysis is the best way of studying the tribological phenomena and understanding the laws of tribology, the relationship between the subject areas is weak if not also considered from a macroscopic perspective.

Furthermore, because tribological problems involve many unique and random properties, and research into tribology lacks a macro and systematical approach, it has been believed that the establishment of a general theory for tribology is difficult or even impossible.

In the present paper, we discuss the extraction of the main influential factors in order to observe and solve the tribological problems using mechanics, with the aim being to obtain more realistic solutions. In our paper, the relationship between the normal pressure and the frictional stress is used to setup the theoretical system of frictional mechanics. Some amount of micro research achievements must therefore be used to complete such a theoretical system of frictional mechanics.

\section{Objects and features studied}

The objects of frictional mechanics are interfaces, each of which consists of a frictional pair. The apparent smooth surface actually has many irregular peaks and valleys when viewed with a microscope, and they are formed during manufacturing processes such as cutting and grinding, because of plastic deformations, vibrations, etc. [23,24]. The micro roughness and 
physical chemistry status of the surfaces are important factors that affect tribological behavior and processes [17]. Beneath the surface, there is a strengthened layer that is several tens of micrometers thick, going from the heavy deformed layer to the light deformed layer. Above them, there is an amorphous or microcrystalline layer that is due to melting, flowing, and then rapid cooling, which takes place during manufacturing. Moreover, the oxidizing layer, adsorbed film, and/or polluted film are on the top. Their common sizes are shown in Fig. 2 in absolute and logarithmic scales.

Based on Fig. 2, we classify the interfaces studied in frictional mechanics as those that are with and without a medium, as shown in Fig. $3[17,25]$.
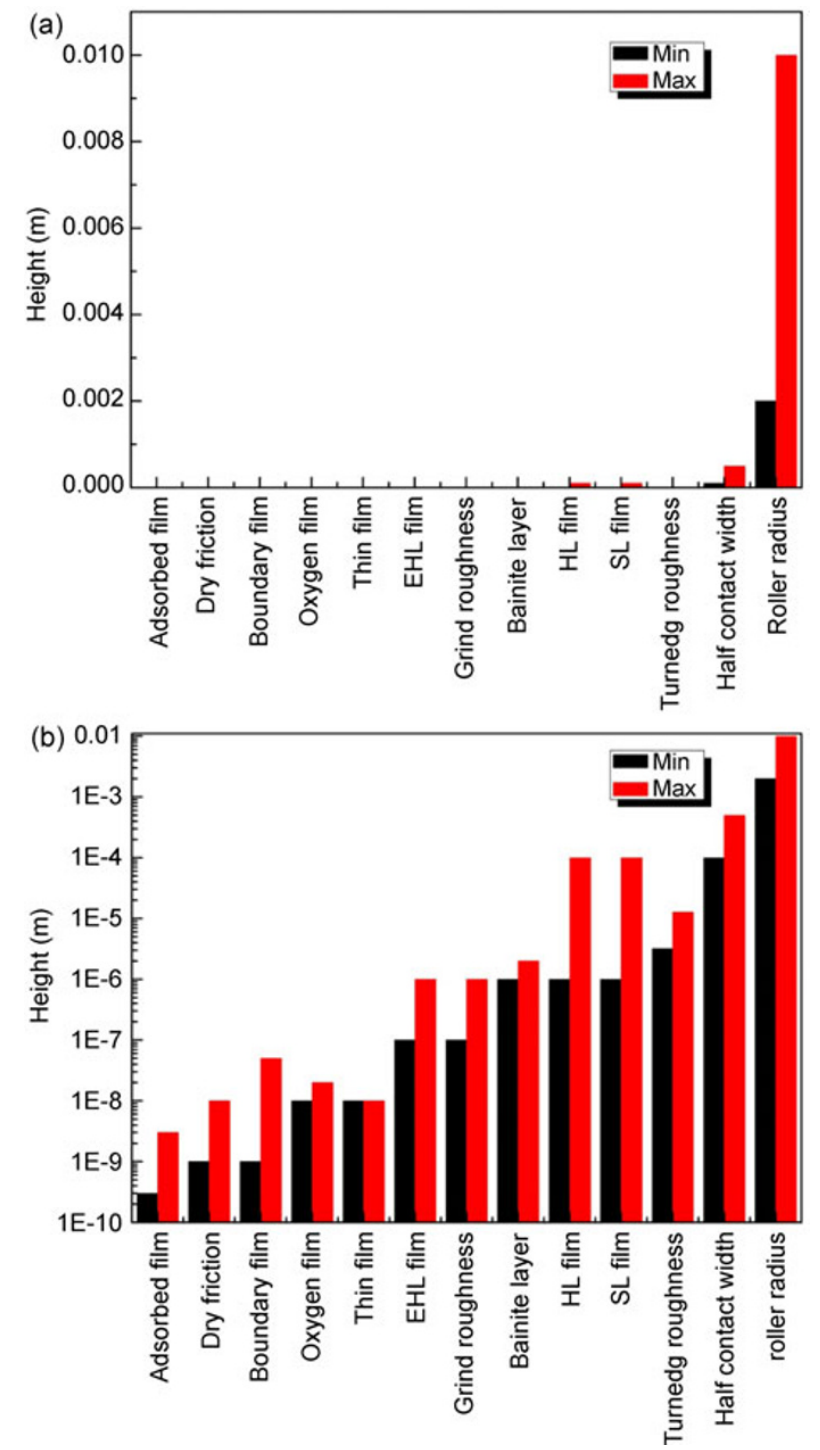

Fig. 2 Comparison of size of surface layers: (a) Common coordinate height; (b) logarithmic coordinate height.

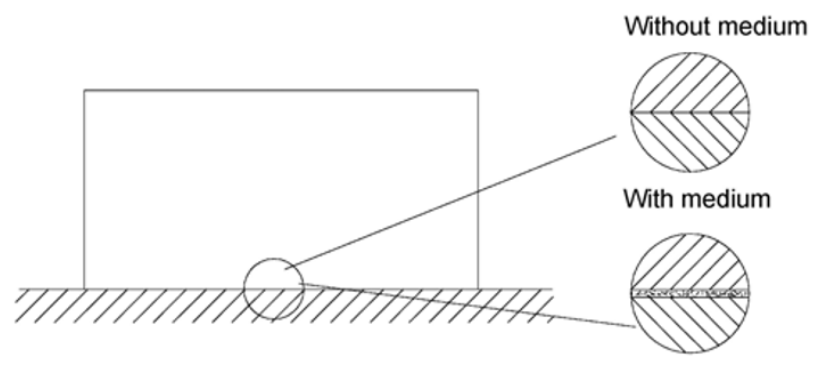

Fig. 3 Interfaces without or with medium.

\section{Basic equations of frictional mechanics}

The purpose of frictional mechanics is to develop the relationship between the frictional stress and the normal pressure on the interface, and to solve their distributions using mechanics motions or equilibrium equations and frictional law.

\subsection{Mechanics equations}

While considering the frictional force, Newton's second law in vector form can be expressed as follows:

$$
\boldsymbol{F}=m a+\boldsymbol{F}_{\mathbf{f}}
$$

where the frictional force $\boldsymbol{F}_{\mathrm{f}}$ cannot be considered as an active force, but as a resistance force induced by the normal load.

For dry friction with motion, Coulomb's law can be written as follows [1]:

$$
F_{\mathrm{f}}=f \mathrm{~N}
$$

where $N$ is the normal load, $F_{\mathrm{f}}$ is the frictional force on the contact surface, and $f$ is the friction coefficient.

Coulomb's law can be defined as the friction coefficient of dry friction, that is, the frictional force is proportional to the normal load. From a macroscopic perspective, it is an empirical formula, but in order to use it on a microscopic level to solve the pressure and frictional stress of the interface, we can write a microCoulomb law as follows:

$$
\tau_{\mathrm{f}}=f p
$$

where $\tau_{\mathrm{f}}$ is the frictional stress, $f$ is the friction coefficient, and $p$ is the normal pressure.

Although Eq. (3) cannot be proven, it is still widely used on a microscopic level. 
For two-dimensional problems, if we set the moving direction as $x$ and the normal direction as $y$, the equilibrium equations for frictional mechanics will be:

$$
\begin{aligned}
& \text { along } x \text { direction: } \quad \int_{x_{1}}^{x_{2}} \tau_{\mathrm{f}}(x) \mathrm{d} x=F \\
& \text { along } y \text { direction: } \quad \int_{x_{1}}^{x_{2}} p(x) \mathrm{d} x=N \\
& \text { to } z \text { axis: } \int_{x_{1}}^{x_{2}} p(x)\left(x-x_{1}\right) \mathrm{d} x=F h+\frac{N\left(x_{2}-x_{1}\right)}{2} \\
& \text { micro Coulomb law: } \tau_{\mathrm{f}}(x)=f p(x)
\end{aligned}
$$

where $x_{1}$ and $x_{2}$ are the two ends of the length of the body in the $x$ direction, $p(x)$ is the normal pressure of the interface, $h$ is the arm of $F$ to Point $x_{1}$, and $\left(x_{2}-x_{1}\right) / 2$ is the arm of the normal load $N$ to Point $x_{1}$.

\subsection{Elastic deformation equation}

The elastic deformation of the surface in the line contact is given as the following equation [26].

$$
v(x)=-\frac{2}{\pi E} \int_{x_{0}}^{x_{e}} p(s) \ln (s-x)^{2} \mathrm{~d} s+c
$$

where $E$ is the equivalent elastic modulus of the materials of the two contact surfaces, $\frac{1}{E}=$ $\frac{1}{2}\left(\frac{1-v_{1}^{2}}{E_{1}}+\frac{1-v_{2}^{2}}{E_{2}}\right), E_{1}$ and $E_{2}$ are the elastic modulus of the materials of the two contact surfaces, respectively, $v_{1}$ and $v_{2}$ are the Poisson's ratios of the materials of the two contact surfaces, respectively, and $c$ is the integral constant to be determined.

For the point contact, the surface elastic deformation can be achieved as follows [26]:

$$
v(x, y)=\frac{2}{\pi E} \iint_{\Omega} \frac{p(s, t)}{\sqrt{(x-s)^{2}+(y-t)^{2}}} \mathrm{~d} s \mathrm{~d} t
$$

\subsection{Equations for interface with fluid medium}

While a medium exists in the interface, the problem with the boundary film formed by the medium can be treated as a dry friction problem. Therefore, no additional mechanics equations are needed. However, if there is a fluid film, Reynolds equation, which is a simple fluid mechanics equation, should be used. The two-dimensional Reynolds equation is given as follows [1]:

$$
\frac{\partial}{\partial x}\left(\frac{\rho h^{3}}{\eta} \frac{\partial p}{\partial x}\right)+\frac{\partial}{\partial y}\left(\frac{\rho h^{3}}{\eta} \frac{\partial p}{\partial y}\right)=6 U \frac{\partial(\rho h)}{\partial x}
$$

where $\rho$ is the density of the lubricant, $h$ is the film thickness, $p$ is the pressure, $\eta$ is the viscosity of the lubricant, $x$ is the coordinate in tangential direction to the surface velocity $U, y$ is the coordinate vertical to the velocity, and $U$ is the surface velocity.

Coulomb's law can no longer be used to obtain the frictional stress between the interface of the solid and fluid, but the constitutive equation of a fluid such as the Newtonian fluid can be used. For a Newtonian fluid, the constitutive equation is given by [1]:

$$
\tau=\eta \frac{\partial u}{\partial z}
$$

where $\tau$ is the shear stress, $z$ is the coordinate across the film thickness, and $u$ is the fluid velocity.

\section{Sliding frictional mechanics}

Generally, the frictional force may not only cause an increase in the shear stress, but it may also change the normal pressure.

\subsection{Slider with no medium}

First, a slider friction problem is used as an example to show the influence of the frictional stress on the normal pressure.

If the slider remains stationary or moves uniformly, the frictional mechanics equations are given by:

$$
\begin{array}{ll}
\text { in } x \text { direction: } & \int_{0}^{l} \tau_{f}(x) \mathrm{d} x=F \\
\text { in } y \text { direction: } & \int_{0}^{l} p(x) \mathrm{d} x=N \\
\text { to } z \text { axis: } & \int_{0}^{l} p(x) x \mathrm{~d} x=F h+\frac{N l}{2}
\end{array}
$$

micro Coulomb's law: $\tau_{\mathrm{f}}(x)=f p(x)$

where $x$ and $y$ are the respective coordinates in the tangential and normal directions to the velocity, $z$ is the axis vertical to the paper, $F$ is the tangential load, $N$ is the normal load, and $l$ is the length of the slider in the $x$ direction.

For convenience, assume that the normal pressure distribution has a linear relationship with the coordinate $x$ : 


$$
p(x)=c+k x
$$

where $c$ and $k$ are the constants to be determined. If they are determined, the normal pressure distribution $p(x)$ and the frictional stress $\tau(x)$ are known.

(1) No friction $(F=0)$ or traction $F$ is in the line of the interface $(h=0)$, as shown in Fig. 4(a). We have:

$$
\begin{aligned}
& p(x)=p_{0}=\frac{N}{l} \\
& \begin{cases}\tau_{\mathrm{f}}(x)=0 & \text { no friction } \\
\tau_{\mathrm{f}}(x)=f p_{0}=f \frac{N}{l} & h=0\end{cases}
\end{aligned}
$$

(2) Small friction $\left(0<F \leqslant \frac{N l}{6 h}\right)$. The normal pressure and the frictional stress are as shown in Fig. 4(b), and are given as:

$$
\begin{aligned}
& p(x)=\frac{N}{l}-\frac{6 F h}{l^{2}}+\frac{12 F h}{l^{3}} x \\
& \tau_{\mathrm{f}}(x)=f\left(\frac{N}{l}-\frac{6 F h}{l^{2}}+\frac{12 F h}{l^{3}} x\right)
\end{aligned}
$$

(3) Large friction $\left(\frac{N l}{6 h}<F \leqslant \frac{N l}{2 h}\right)$. Some parts of the slider have no normal pressure or frictional stress, as shown in Fig. 4(c).

$$
\begin{gathered}
p(x)= \begin{cases}0 & 0 \leqslant x<b \\
\frac{(x-b)}{(l-b)} p_{2} & b \leqslant x \leqslant l\end{cases} \\
\tau_{\mathrm{f}}(x)= \begin{cases}0 & 0 \leqslant x<b \\
f \frac{(x-b)}{(l-b)} p_{2} & b \leqslant x \leqslant l\end{cases}
\end{gathered}
$$

where $b=\frac{5 l}{2}-\frac{3 h F}{N} ;$ and $p_{2}=\frac{4 N^{2}}{6 h F-3 l N}$

(4) Critical situation $\left(F=\frac{N l}{2 h}\right)$. With the exception of the front end of the slider, there is no normal pressure or frictional stress on the other parts of the interface, as shown in Fig. 4(d).

(5) Turnover situation $\left(F>\frac{N l}{2 h}\right)$. The object cannot move uniformly or be balanced.

An application of the results above is the wedge (a) $Y$

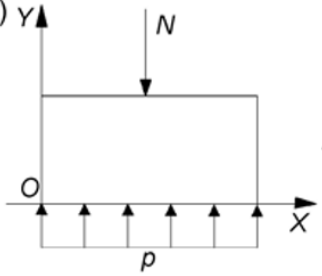

(c)

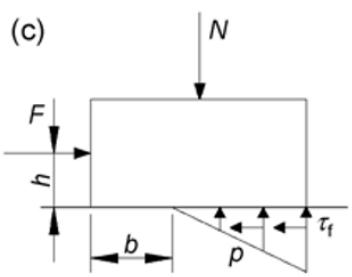

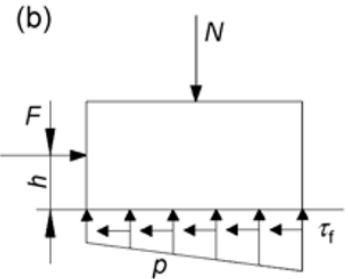

(d)

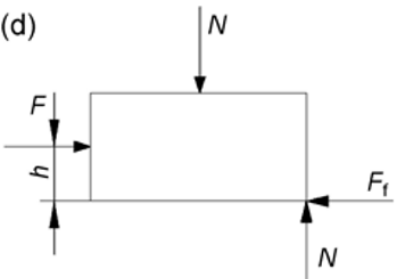

Fig. 4 Frictional stress and pressure distribution when considering friction: (a) No friction; (b) small friction; (c) large friction; (d) critical status.

key in the design of mechanical elements [27, 28]. After installation, the working surfaces of the wedge key are the upper and lower ones, as shown in Fig. 5(a). Without the rotation, there is only a normal load $N$ acting on the working surfaces, as shown in Fig. $5(b)$, where $p_{0}=N / l b, l$ is the length of the key, and $b$ is the width. When rotating with a torque, a frictional force is produced to resist the torque because there is a relative motion between the shaft and the hub [28]. This tendency produces a micro twist deformation so that the pressure changes along the length and width of the key. The resultant $N$ of the pressure no longer passes the center of the shaft, but is at a distance of $x_{0}$, as shown in Fig. 5(c).

For convenience, consider the key and the shaft as one body, and the resultant force of the bottom pressure of the cylinder is substituted by $N$. If we assume that the pressure is linearly proportional to the length of the key, it will have a triangular distribution, as shown in Fig. 5(c), where $x_{0} \approx b / 6$ and $y_{0} \approx d / 2[27,28]$. We can write the balance equation to the shaft center as:

$$
T=N x_{0}+f N y_{0}+f N d / 2
$$

where $T$ is the torque, $f$ is the friction coefficient, and $d$ is the diameter of the shaft.

Then, the resultant force of the pressure is as follows:

$$
N=\frac{T}{x_{0}+f y_{0}+f d / 2}=\frac{6 T}{b+6 f d}
$$

The maximum pressure $p_{\max }$ is on the right end of 

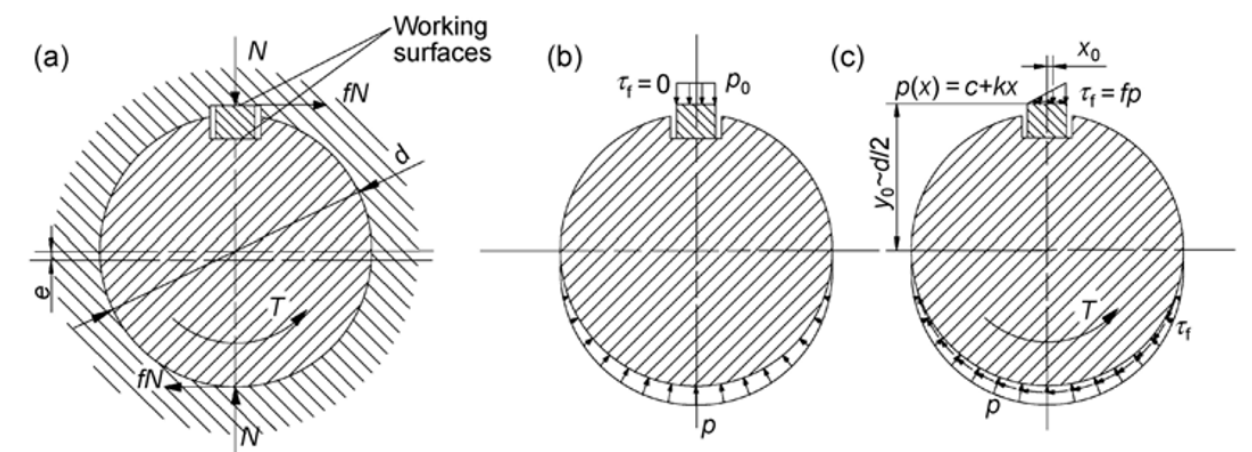

Fig. 5 Interface stress analysis of wedge key [27, 28]: (a) loads on wedge key; (b) before rotation; (c) rotation.

the key, which is double the average pressure $p_{0}$, and the frictional stress can also be obtained.

$$
\begin{aligned}
& p_{\text {max }}=2 p_{0}=\frac{2 N}{b l}=\frac{2 T}{\left(x_{0}+f y_{0}+f d / 2\right) b l}=\frac{12 T}{(b+6 f d) b l} \\
& \tau_{\text {f max }}=f p_{\text {max }}=\frac{12 T f}{(b+6 f d) b l}
\end{aligned}
$$

\subsection{Slider with medium}

Three types of slider with the medium are shown in Fig. $6[27,28]$.

(a) Two parallel surfaces: if we do not consider the hydrostatic situation, the medium can be only the boundary film, that is, boundary lubrication. Because the frictional force is very small, the normal pressure distribution is hardly influenced by the medium. Therefore, the normal pressure can be considered to be uniform, as shown in the lower figure of Fig. 6(a).

Therefore, the frictional stress of the boundary lubrication obeys Coulomb's law in Eq. (3). Because the pressure is uniform, the frictional stress of the
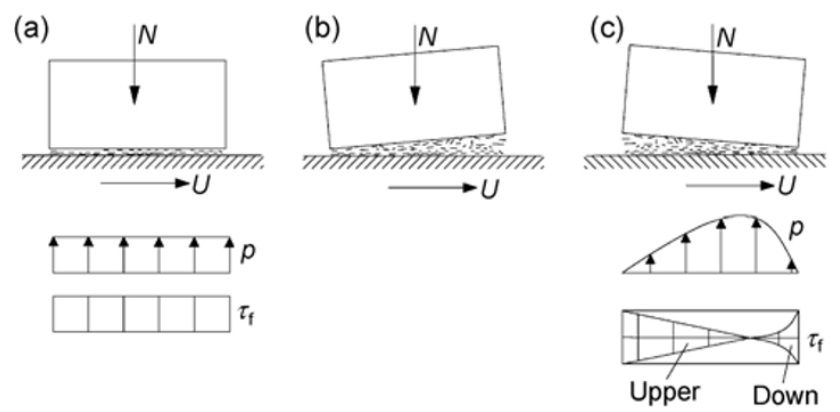

Fig. 6 Pressure and frictional stress of slider with inter-medium: (a) Parallel; (b) divergent; (c) convergent. parallel slider is also uniform. However, the difference with the dry friction is that the friction coefficient of the boundary lubrication relates to the strength of the boundary film, but it is much smaller than the dry friction coefficient.

(b) Divergent wedge: it cannot exist stably in practice, but quickly changes to Case (a) or (c).

(c) Convergent wedge: this is the hydrodynamic lubrication status. The pressure distribution can be obtained to solve Reynolds equation, as shown in the lower figure of Fig. 6(c). The expression for the pressure is as follows [26]:

$$
p=-\frac{6 U \eta l}{k h_{1}}\left(-\frac{1}{h}+\frac{h_{1} h_{2}}{h_{1}+h_{2}} \frac{1}{h^{2}}+\frac{1}{h_{1}+h_{2}}\right)
$$

where $k=\frac{h_{2}-h_{1}}{h_{1}}, h$ is the film thickness, $h_{1}$ is the film thickness of the inlet, $h_{2}$ is the film thickness of the outlet, and $l$ is the length of the slider.

Its frictional stress cannot be obtained from Coulomb's law in Eq. (3), but can be obtained from the constitutive equation in Eq. (8). For any point $x$, the frictional stress on the upper and lower interfaces are:

$$
\tau_{\mathrm{h}, 0}= \pm \frac{\partial p}{\partial x} \frac{h}{2}+\eta \frac{v}{h}
$$

where $\tau_{0}$ and $\tau_{\mathrm{h}}$ are the shear stress (frictional stress) of the upper and lower surfaces, respectively.

In Fig. 7, a dimensionless solution of pressure $P$, film thickness $H$, lower interface frictional stress $\tau_{0}$, and upper interface frictional stress $\tau_{\mathrm{h}}$ are given for the slider with the medium [26]. It clearly shows that the pressure and frictional stress with the medium are very different from those in the case of dry friction. 


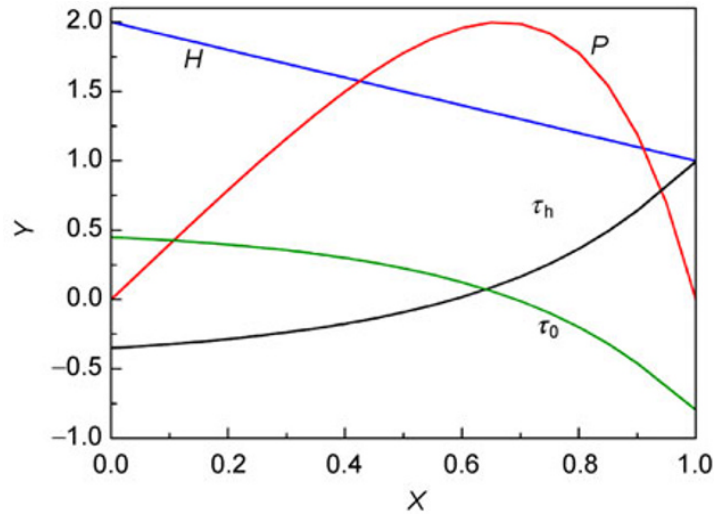

Fig. 7 Pressure, film thickness, and frictional stresses of up and down interfaces.

\subsection{Journal bearing (hydrodynamic lubrication)}

For a journal bearing with hydrodynamic lubrication, the bearing was extended along the circumference, and Reynolds equation Eq. (6) should be expressed by the cylindrical coordinates $[5,6]$, that is:

$$
\frac{\partial}{\partial \theta}\left(\frac{\rho h^{3}}{\eta} \frac{\partial p}{\partial \theta}\right)+\frac{\partial}{\partial y}\left(\frac{\rho h^{3}}{\eta} \frac{\partial p}{\partial y}\right)=6 R \frac{\partial(U \rho h)}{\partial \theta}
$$

where $\theta$ is the angular velocity coordinate, $R$ is the radius of the journal bearing, and $h$ is the film thickness (the shape of the clearance) [28]:

$$
h=e \cos \theta+c=c(1+\varepsilon \cos \theta)
$$

where $e$ is the eccentricity, $c$ is the clearance, $\varepsilon=e / c$ is the eccentricity ratio, and $\theta$ is the circumferential coordinate starting from the maximum film thickness.

If there is no rotation, the pressure is shown as in Fig. 8(a) [28]. While there is a rotation, the hydrodynamic effect must be considered so that a hydrodynamic lubrication film will be formed. By solving
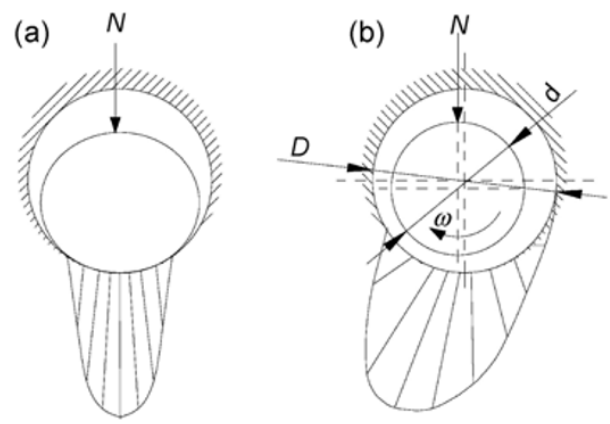

Fig. 8 Pressure distribution of journal bearing: (a) Without rotation; (b) with rotation.
Reynolds equation in Eq. (16), the pressure distribution can be obtained, as shown in Fig. 8(b) [28]. Compared with the two figures of Fig. 8, it can be seen that the medium significantly influences the interface pressure. The phenomenon where the pressure deviates from the original signifies that the solution of the hydrodynamic solution is a deviation of the nonhydrodynamic one.

\section{Rolling frictional mechanics}

\subsection{Rolling friction without medium}

Depending on the actions and friction mechanics, rolling can be divided into four types: (1) Free rolling; (2) traction pure rolling; (3) torque driven rolling; and (4) sliding-rolling, as shown in Fig. 9.

First, we discuss the frictional mechanics of rigid rolling. For rigid rolling, the normal pressure $p$ and the frictional stress tend to infinity because the contact area of the line and point is zero [29]. However, the total normal load and frictional force have limited values.

\subsubsection{Free rolling}

Free rolling means that there is no driving force or torque. In other words, there is no relative motion between the moving pair at the contact point. If the
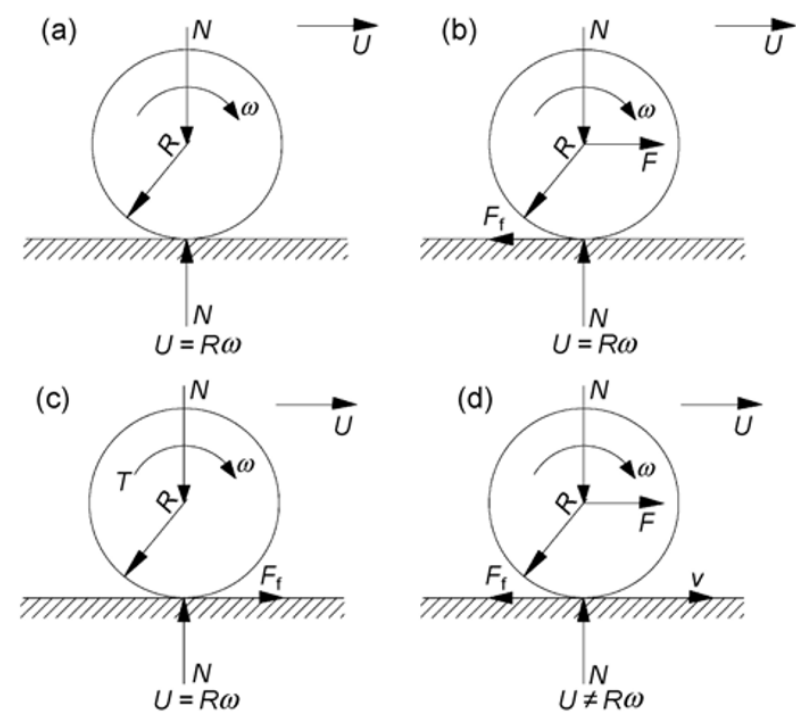

Fig. 9 Types of rolling: (a) Free rolling; (b) traction rolling; (c) torque driven; (d) sliding-rolling. 
external resistance is not considered, such as air resistance, there is no friction at the contact point, that is, [29]:

$$
\begin{aligned}
p & =\frac{N}{A} \longrightarrow \infty \\
\tau_{\mathrm{f}} & =0
\end{aligned}
$$

It should be noted that Eq. (18) is only suitable in the ideal case. In reality, the frictional force is not equal to zero because of the external resistance.

Ideal status: It is generally believed that for rolling, there must be some frictional force at the contact point. However, the frictional force is a force that resists the motion or motion tendency, while the contact point is the instant center of motion of the object so that its velocity is equal to zero. Because there is no tendency to move, there is no frictional force on the contact point. It should be noted that although there is no frictional force, free rolling can occur. Furthermore, the absence of a frictional force means that the surface is smooth. However, because of external resistances, such as air resistance, there will be a frictional force that balances the resistance. Also, because there is a resistance, it will slow down the rolling of the wheel until the rotational speed becomes zero.

\subsubsection{Traction pure rolling}

It should also be noted that the pure rolling driven by a traction $F$ may have two rolling types, as shown in Fig. 10. Because the traction is greater or less than the total resistance, the rolling accelerates or decelerates, respectively, and as the traction becomes equals to the total resistance, the rolling speed becomes constant [29].

A traction pure rolling has no relative velocity at the contact point driven by an outside traction to

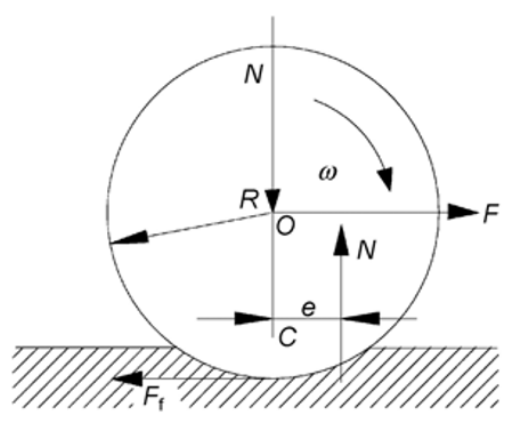

Fig. 10 Rolling friction coefficient. have the frictional pair to roll. From the viewpoint of frictional mechanics, because there is no relative motion, the frictional force is less than the maximum statics frictional force.

$$
F_{\mathrm{f}}=F<F_{\mathrm{f} \max }
$$

The rolling friction coefficient is defined as the ratio of the rolling frictional moment to the normal load, that is,

$$
k=\frac{F R}{N}=e
$$

where $k$ is the rolling frictional coefficient. The dimensional rolling friction coefficient $k$ is the length.

\subsubsection{Pure rolling driven by torque}

Pure rolling driven by a torque is the case where there is rolling at the contact point having no relative velocity. Such a motion is similar to two friction wheels rolling with no sliding. Whether a car rides or a person walks on the ground is in this way to move, that is, one wheel is the car's wheel or the person's legs (radius) and feet (wheel rim), and another wheel is the earth.

From the perspective of frictional mechanics, if there is no sliding, there is no relative motion at the contact point. Therefore, the friction is a static friction, and the frictional force at the contact point is smaller than the maximum frictional force of the contact surface.

$$
F_{\mathrm{f}}<F_{\mathrm{f} \max }=f_{\text {max }} N
$$

where $f_{\max }$ is the maximum static frictional coefficient.

\subsubsection{Sliding-rolling}

Sliding-rolling can be considered to be a combination of sliding and pure rolling, as shown in Fig. 9(d). When Eq. (21) is not satisfied, the wheel appears to slip to the ground surface so that [29]:

$$
F \geqslant F_{\text {fmax }}
$$

Therefore, there is a relative motion at the contact point so that both sliding and rolling exist simultaneously. 


\subsection{Rolling under elastic deformation}

The elastic deformation of a cylinder or a ball making contact with a rigid plane can be obtained by Hertzian contact theory [20,30]. Such an elastic deformation and the corresponding normal pressure are shown in Fig. 11.

$$
\begin{aligned}
& p=p_{\mathrm{H}} \sqrt{1-\left(\frac{x}{b}\right)^{2}} \\
& \tau_{\mathrm{f}}=f p=f p_{\mathrm{H}} \sqrt{1-\left(\frac{x}{b}\right)^{2}}
\end{aligned}
$$

where $p_{\mathrm{H}}$ is the maximum Hertzian pressure and $b$ is the half-width of the contact region.

Applying the micro Coulomb law in Eq. (3) as the relationship between the frictional stress and the normal pressure, and after analyzing the sliding of elastic bodies, Bufler concluded that if only the two elastic constants (the shear modulus $G$ and Poisson's ratio $v$ ) of the contact bodies are the same, the frictional stress does not affect the normal pressure [31].

The analysis made by Johnson [29] shows that if the two materials are different, the frictional stress will change the distribution of the normal pressure and the contact width, as shown in Fig. 12. While the friction coefficient $f=0.1$, the elastic coefficient $\beta=\frac{1}{2}\left\{\frac{\left[\left(1-2 v_{1}\right) / G_{1}\right]-\left[\left(1-2 v_{2}\right) / G_{2}\right]}{\left[\left(1-v_{1}\right) / G_{1}\right]+\left[\left(1-v_{2}\right) / G_{2}\right]}\right\}=0.2$, the center $x_{0}$ of the normal pressure will move by about $0.12 a$, and the width of the contact region will increase by about $0.08 \%$.

Johnson's conclusion was that because the friction coefficient exceeds 1.0 and the combination coefficient $\beta$ of most materials does not exceed 0.21 , the influence of the frictional stress on the contact pressure distribution and movement of its center are negligible.

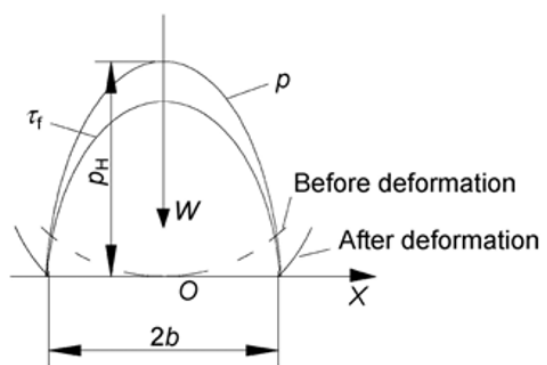

Fig. 11 Hertzian stress and deformation in line contact.

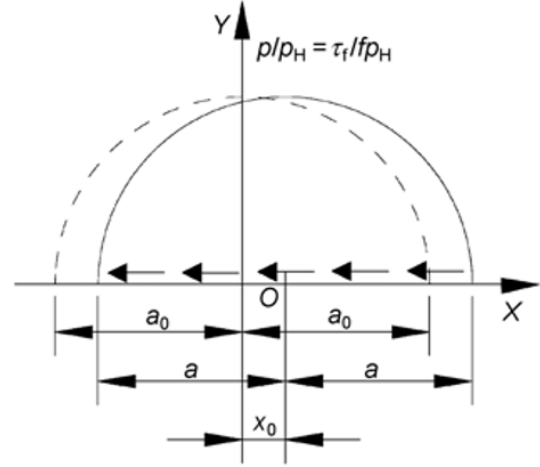

Fig. 12 Influence of elastic constant to pressure distribution of sliding cylinder [29].

\subsection{Rolling friction with medium (elastohy- drodynamic lubrication)}

If we do not consider the friction, the contact pressure can be solved by Hertzian contact theory [29-31]. For the line contact, the contact pressure is shown in Fig. 11. While there is a lubricant, the effect of friction must be considered, such as in gears and rolling contact bearings, etc. The pressure can be obtained by solving Reynolds equation in Eq. (7) and the elastic deformation in Eq. (5) or (6) together, which is the EHL solution. The non-dimensionless pressure $P$, film thickness $H$, and shearing stresses $\tau_{0}$ and $\tau_{\mathrm{h}}$ are shown in Fig. 13, which is a typical EHL solution for a line contact. The pressure is not the same as the Hertzian stress, but it extends to outside the inlet, and the second pressure peak can be found at the outlet region, where a corresponding necking also appears [32-35].

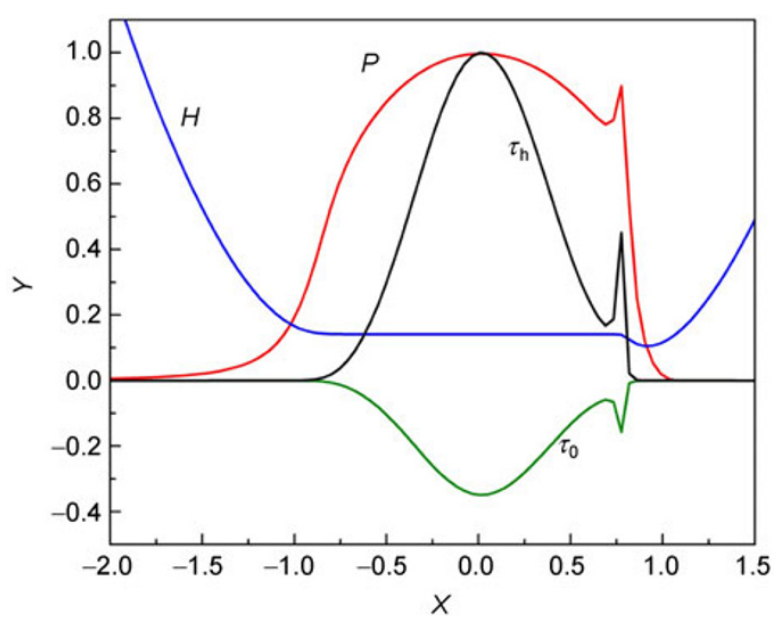

Fig. 13 Pressure and film thickness of EHL on line contact [26]. 


\subsection{Transverse elastic rolling-sliding without medium (elastic sliding of belt transmission)}

The pressure and the frictional stress of the belt transmission are based on the Euler equation for flexible bodies. It can be expressed as follows [27, 28]:

$$
T=T_{2} e^{f \theta}
$$

where $T$ is the tension of the contact arc to the angle $\theta$, $T_{2}$ is the tension of the loose side, and $f$ is the friction coefficient.

With the force balance conditions, the normal load of the belt transmission is equal to [28]:

$$
N=\frac{T_{2}}{f} e^{f \theta}
$$

where $N$ is the normal load and $\theta$ starts from the point at which the loose side belt enters the wheel.

To derive Eq. (25) for angle $\theta$, we use the following pressure distribution.

$$
p=\frac{T_{2}}{R} e^{f \theta}
$$

where $R$ is the radius of the wheel and $p$ is the normal pressure.

Because the pressure is related to the radius $R$ of the belt wheel, the pressure on the smaller belt wheel $R_{1}$ is larger than that on the larger belt wheel. Using Coulomb's law in Eq. (3), we can obtain the following frictional stress from Eq. (26).

$$
\tau_{\mathrm{f}}=\frac{T_{2} f}{R} e^{f \theta}
$$

where $\tau_{\mathrm{f}}$ is the frictional stress.
Figure 14(a) shows the total stresses on the belt transmission, while the tension distribution according to Eq. (24) and the elastic sliding arcs on the smaller and larger wheels are illustrated in Fig. 14(b) [28].

The analysis of the sliding-rolling of the belt is as follows [29]. From Eq. (23), it is known that the extension of the belt is different because it has a different tension, but the wheels have no deformation. Therefore, as the belt passes the belt wheels, it appears to be sliding because the extension varies gradually. This is called "elastic sliding". The tension strains of the loose and tension sides have the following relationship.

$$
\varepsilon_{1}=\lambda T_{1}>\varepsilon_{2}=\lambda T_{2}
$$

where $\lambda$ is the tension ratio and $\varepsilon$ is the tension strain.

While the belt works normally, the elastic sliding only occurs in the area of the contact arcs, where the belt leaves the wheels [28], as shown in Fig. 14(b). This is because the differences in the velocity of the belt and wheels are the maximum. Therefore, elastic sliding starts from these two points. By using Eq. (28), the following elastic sliding ratio can be obtained.

$$
\xi=\lambda\left(T_{1}-T_{2}\right)
$$

The contact arc at which there is no elastic sliding is called the static arc. As the transmission power increases, the elastic sliding arc will also increase. As the elastic sliding region extends to the whole contact arc, the total frictional force reaches a maximum value. After that, if the power continues to increase, there will be significant sliding between the wheel and the belt, that is, the slip and belt transmission will fail to work.

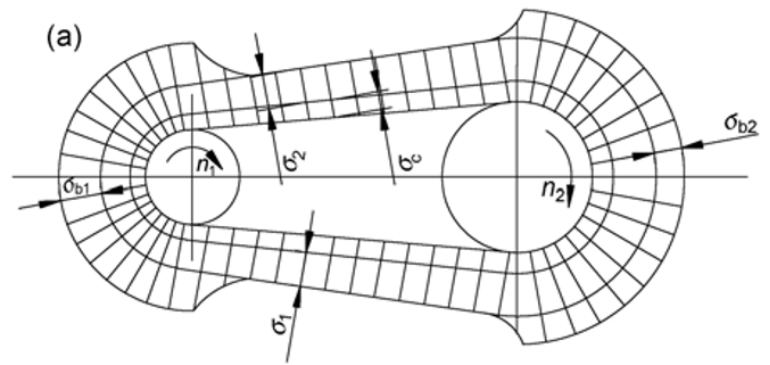

(b)

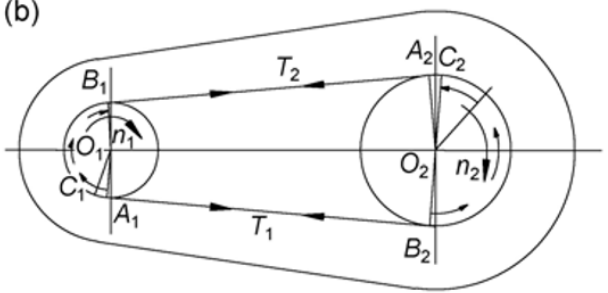

Fig. 14 Force on transmission belt [27, 28]: (a) All stresses; (b) tensions and elastic sliding arcs. 


\section{Discussions}

Friction is the primary cause of the tangential force that exists on an interface. Whether it is a sliding, rolling, or sliding-rolling motion, friction will exist on an interface and produce a frictional force. The frictional force that exists during movement can usually be determined by measuring the friction coefficient. Although in engineering, it is useful to calculate the frictional force using the frictional coefficient, the final tangential force should still be determined by balancing the external forces.

Besides, there is also a frictional force in a stationary interface, that is, a static frictional force. A static frictional force varies from zero to its maximum value according to the magnitude of an external force. Therefore, the determination of the tangential force is also based on balancing the external forces.

Now, we list all of the results above in Table 1 to clarify the difference between cases involving friction and non-friction, deformation and non-deformation, and with and without a lubricant. It can be seen that:

(1) There are differences in the results for cases that both consider and do not consider friction;

(2) The normal pressure and the frictional stress influence each other;

(3) If there is a lubricant, the effect of fluid-solid coupling is significant.

Table 1 Comparison of pressure and frictional stress under different working conditions.

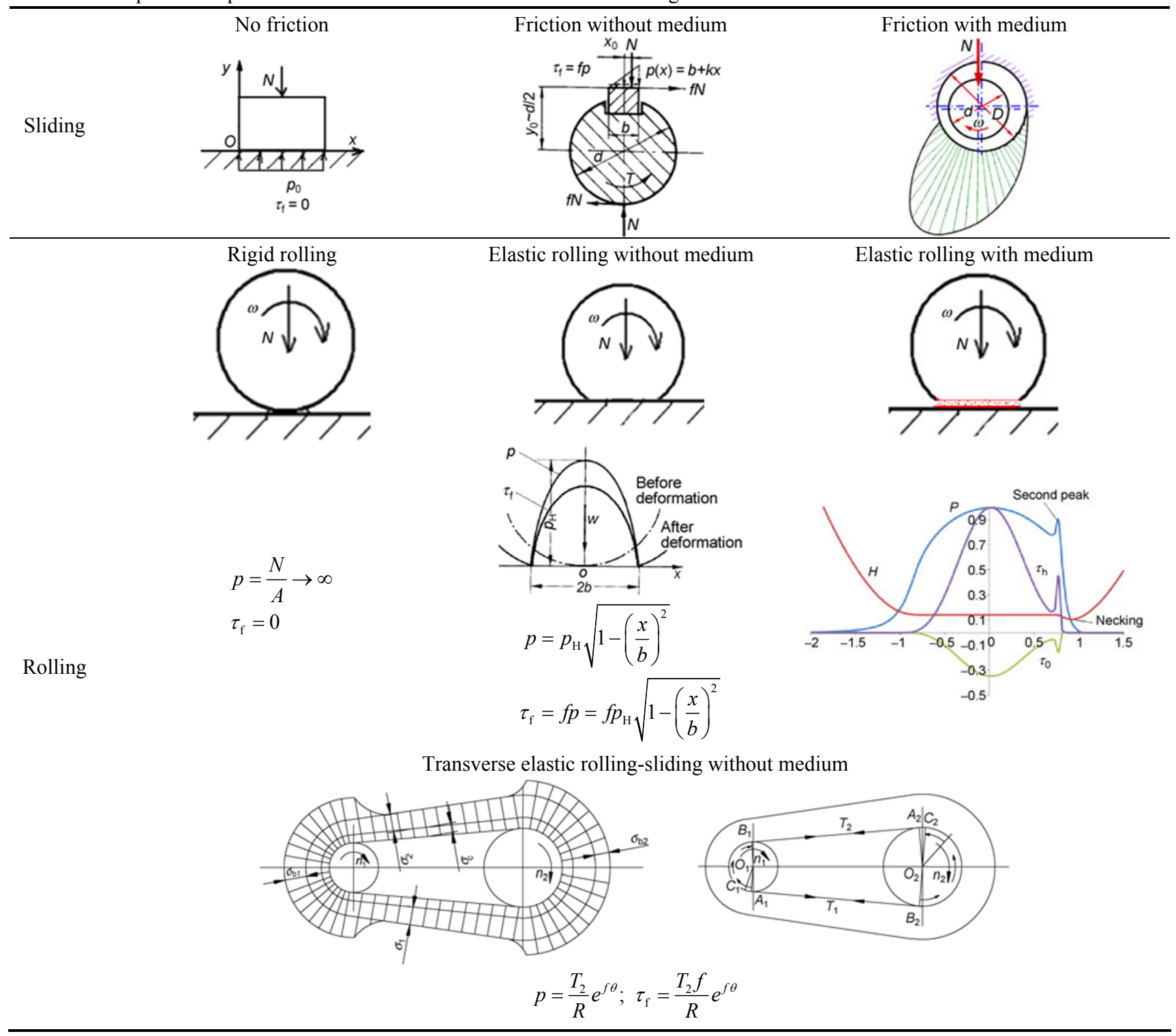


The frictional force is usually considered to be in the direction opposite to the moving direction. For the sliding motion, the frictional force can be easily determined, and it is along the tangent direction of the interface. However, for the rolling motion, the frictional force may be the same as the direction of motion. Most research has focused on determining the influences of different factors on the tribology phenomenon on a microscopic level. The establishment of frictional mechanics theory requires the synthesis of more microscopic studies that are combined with results obtained macroscopically. It is believed that this can contribute to the transition of tribology from an experiential subject to an analytical subject.

\section{Conclusions}

Based on the discussion and analysis above, we presented a set of basic equations of frictional mechanics. Relationships such as Coulomb's law, which exist between the frictional force and the normal pressure, were added to the general mechanics equations without considering friction. By comparing cases involving sliding and non-sliding and with and without lubricants, the following conclusions were made:

(1) The frictional mechanics deviates from the classical mechanics problem that does not consider friction, that is, the solution of a frictional mechanics problem can be considered to be a variant of the classical mechanics solution. While the frictional force or friction coefficient tends to zero, the solution becomes the original one.

(2) The frictional force and the normal load influence each other, which means that the normal pressure and the frictional stress depend on each other. In order to solve frictional problems, the equations must therefore be solved together. If we simply superimpose the results for the cases with and without friction, there may be significant errors.

\section{Acknowledgements:}

The project was supported by the National Natural Science Foundation of China (Grant No. 51175182).

Open Access: This article is distributed under the terms of the Creative Commons Attribution License which permits any use, distribution, and reproduction in any medium, provided the original author(s) and source are credited.

\section{References}

[1] Wen S Z, Huang P. Principles of Tribology. Singapore: Weley and Tsinghua University Press, 2011.

[2] Bhushan B. Introduction to Tribology. New York (USA): Wiley, 2002.

[3] Moore D F. Principles and Applications of Tribology. London (UK): Pergamon Press, 1975.

[4] Quan Y X. Engineering Tribology. Hangzhou (China): Zhejiang University Press, 1994.

[5] Huang P, Meng Y G, Xu H. Tribology Course. Beijing (China): Higher Education Press, 2008.

[6] Pinkus O, Sternlicht B. Theory of Hydrodynamic Lubrication. London: McGraw-Hill, 1961.

[7] Крагельский И В (Wang Y L, et al., translate). Principles of Friction and Wear. Beijing (China): Mechanical Industry Press, 1982.

[8] Bowden F P, Tabor D. The Friction and Lubrication of Solid. Oxford (UK): Oxford University Press, 1954.

[9] Nosonovsky M, Bhushan B. Multiscale friction mechanisms and hierarchical surfaces in nano- and bio-tribology. Mater Sci Eng R Rep 58(3-5): 162-193 (2007)

[10] Kapsa P. Tribology at different scales. Adv Eng Mater 3(8): 531-537(2001)

[11] Xu Z M, Huang P. Study on the energy dissipation mechanism of atomic-scale friction with composite oscillator model. Wear 262(7-8): 972-977 (2007)

[12] Kim H J, Kim D E. Nano-scale friction: A review. Int $J$ Precis Eng Manuf 10(2): 141-151 (2009)

[13] Williams J A, Le H R. Tribology and MEMS. J Phys D: Appl Phys 39(12): R201-R214 (2006)

[14] Falvo M R, Superfine R. Mechanics and friction at the nanometer scale. J Nanopart Res 2(3): 237-248 (2000)

[15] Fujisawa S, Ando Y, Enomoto Y. Microscale friction and surface force. J Jpn Soc Tribologis 44(6): 409-413 (1999)

[16] Wen S Z, Huang P, et al. Interface Science and Technology. Beijing (China): Tsinghua University Press, 2011.

[17] Martin J M, Matta C, Bouchet M I D B, Forest C, Mogne T L, Dubois T, Mazarin M. Mechanism of friction reduction of unsaturated fatty acids as additives in diesel fuels. Friction 1(3): 252-258(2013)

[18] Samadashvili N, Reischl B, Hynninen T, Ala-Nissilä T, Foster A S. Atomistic simulations of friction at an ice-ice interface. Friction 1(3): 242-251(2013) 
[19] Hu Y, Ma T, Wang H. Energy dissipation in atomic-scale friction. Friction 1(1): 24-40(2013)

[20] Du Q H, Yu S W, Yao Z H. Theory of Elasticity. Beijing (China): Science Press, 1986.

[21] Johnson K L, Kendall K, Roberts A D. Surface energy and the contact of elastic solids. Proc $R$ Soc Lond A 324(1558): 301-313 (1971)

[22] Derjaguin B V, Muller V M, Toprov Y P. Effect of contact deformations on the adhesion of particles. J Colloid Interf Sci 67: 314-326 (1975)

[23] Huang P, Lai T M. Friction model based on real contact area (in Chinese). J South China Univers Tech 40(10): 109114 (2012)

[24] Greenwood J A, Williamson J B. Contact of nominally flat surface. Proc R Soc Lond A 295: 300-319 (1966)

[25] Xu J Q. Interface Mechanics. Beijing (China): Science Press, 2011.

[26] Huang P. Lubrication Numerical Calculation Methods. Beijing (China): Higher Education Press, 2012.

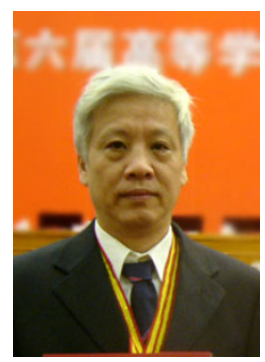

Ping HUANG. He received his Ph.D degree in mechanical engineering from Tsinghua University, Beijing, China in 1989. He worked at the State Key Laboratory of Tribology at Tsinghua from 1989 to 1996, and joined the Mechanical

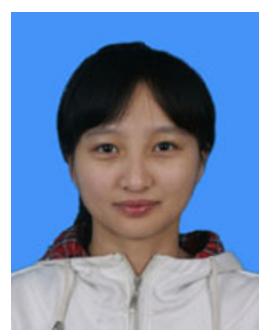

Qianqian YANG. She received her Bachelor degree in mechanical engineering in 2011 from South China University of Technology,
[27] Pu L G, Ji M G. Mechanical Design (Sixth Edition). Beijing (China): Higher Education Press, 1996.

[28] Huang P, Zhu W J. Course of Mechanical Design. Beijing (China): Tinghua University Press, 2012.

[29] Johnson K L. Contact Mechanics. Cambridge (UK): Cambridge University Press, 1985.

[30] Stephen T, Goodier J N. Theory of Elasticity. New York (USA): McGraw-Hill book Company, 1951.

[31] Bufler H Z. Theorie der rollenden Reibung. Ingenieur Archiv 27: 137-140 (1959)

[32] Dowson D, Higginson G R. Elasto-hydrodynamic Lubrication. London (UK): Pergamon Press, 1997.

[33] Wen S Z, Yang P R. Elasto-Hydrodynamic Lubrication. Beijing (China): Tsinghua University Press, 1992.

[34] Johnson K L, Tevaarwerk J L. Shear behavior of elastohydrodynamic oil films. Proc R Soc Lond A 356: 215-236 (1977)

[35] Bair S, Winer W O. Rheological model for elastohydrodynamic contacts based on primary laboratory data. $J$ Lubr Technol 101: 258-265 (1979)

Design and Theory at South China University of Technology since then. His current position is a professor of the laboratory. His research areas cover the measurements and numerical calculations of different lubrication conditions and the mechanism of interfacial micro-friction.

Guangzhou, China. After then, she is a Ph.D candidate in mechanical design and theory at the same university. Her research area is mainly about non-Newtonian fluid lubrication. 